\title{
Characterization of Rolling Contact Fatigue Cracks in Rails by Eddy Current Pulsed Thermography
}

DOI:

10.1109/TII.2020.3003335

Document Version

Accepted author manuscript

Link to publication record in Manchester Research Explorer

Citation for published version (APA):

Zhu, J., Withers, P., Wu, J., Liu, F., Yi, Q., \& Tian, G. (2020). Characterization of Rolling Contact Fatigue Cracks in Rails by Eddy Current Pulsed Thermography. IEEE Transactions on Industrial Informatics, 17(4).

https://doi.org/10.1109/TII.2020.3003335

\section{Published in:}

IEEE Transactions on Industrial Informatics

\section{Citing this paper}

Please note that where the full-text provided on Manchester Research Explorer is the Author Accepted Manuscript or Proof version this may differ from the final Published version. If citing, it is advised that you check and use the publisher's definitive version.

\section{General rights}

Copyright and moral rights for the publications made accessible in the Research Explorer are retained by the authors and/or other copyright owners and it is a condition of accessing publications that users recognise and abide by the legal requirements associated with these rights.

\section{Takedown policy}

If you believe that this document breaches copyright please refer to the University of Manchester's Takedown Procedures [http://man.ac.uk/04Y6Bo] or contact uml.scholarlycommunications@manchester.ac.uk providing relevant details, so we can investigate your claim.

\section{OPEN ACCESS}




\title{
Characterization of Rolling Contact Fatigue Cracks in Rails by Eddy Current Pulsed Thermography
}

\author{
Junzhen Zhu, Student Member, IEEE, Philip John Withers, Jianbo Wu, Member, IEEE, Feng Liu, \\ Qiuji Yi, Zijun Wang, and Gui Yun Tian, Senior Member, IEEE
}

\begin{abstract}
Locating and characterizing rolling contact fatigue ( $R C F)$ cracks in rails is gaining attention in the railway industry. Eddy current pulsed thermography (ECPT) can detect such cracks by combining the benefits of electromagnetic excitation and thermal diffusion. To date, most studies focus on investigating specific features based on artificial defects and verification on real cracks is lacking. To establish the best means of characterizing cracks of different inclination angles, eight spatial and temporal ECPT features are evaluated on idealized and real RCF cracks. Results show that longer time slots and pulse durations make the relations clearer. For evaluation of real RCF cracks, the area-based and the kurtosis-based features are the most suitable and robust measures for characterizing inclination angles.
\end{abstract}

Index Terms-Railway rails, rolling contact fatigue cracks, inclination angle characterization, eddy current pulsed thermography.

\section{INTRODUCTION}

$\mathrm{R}$ OLLING contact fatigue (RCF) defects have become one of the major threats to the railway transport safety [1]. They are a group of flaws that include RCF cracks, spalling, shelling, squats, etc. RCF cracks are also known as head checks or gauge corner cracking when they occur at the rail gauge corner. They are induced by repeated high-contact stresses between wheel and rail arising from traction, braking and steering. RCF cracks can be considered as a type of surface-breaking crack. In contrast to stress corrosion cracking [2], RCF cracks in the initial and intermediate stages tend to be clustered as fish scales and propagate downward at specific inclination angles [3], [4]. Apart from readily locating RCF cracks, the challenge is to characterize the geometric parameters, e.g., inclination angle, pocket length, crack opening, of RCF cracks. Recently, the suitability of several nondestructive testing (NDT) techniques has been investigated [5], [6]. Still, it is challenging to characterize the parameters of RCF cracks because of their clustered distributions and geometric interactions. Compared with the above-mentioned NDT techniques, infrared thermography (IRT) with the advantage of non-contact sensing and easy visualization of tiny thermal variations is gaining increasing attention [7]-[13]. IRT

This work is supported in part by the Engineering and Physical Sciences Research Council under Grant EP/J012343/1 and National Natural Science Foundation of China under Grant 61527803, 51875576, 51505308, and 61905038 . methods can be divided into active IRT and passive IRT depending on whether they are externally stimulated or self-radiating. With the advantage of fast testing and high SNR, active IRT is increasingly used in a wide range of applications [14]-[16]. As a promising candidate, eddy current pulsed thermography (ECPT), also known as induction thermography and electromagnetic thermography, exploits electromagnetic excitation and thermal diffusion. These enable ECPT to easily heat the clustered defects and characterize their geometric parameters [17]-[19]. For quantifying angular defects using the ECPT technique, Abidin et al. [20] extracted the inclination of the line scan from the transient thermal distribution and the maximum temperature to quantify the slot angle and pocket length, respectively. Oswald-Tranta [21] used Fourier transformation to investigate the relation between the phase contrast and the inclination angle for both non-magnetic and ferro-magnetic materials. Liu et al. [22] unitized skewness- and kurtosis-based spatial features to characterize inclination angles. Unfortunately, most of the above investigations are based on testing man-made specimens and lack verification on real cracks. Additionally, many studies focus primarily on inclination angles without fully discussing the influences of other variables, e.g., pocket length and crack opening.

Previous work has studied pocket length characterization [23], [24]. To compare the capabilities of different ECPT features for characterizing inclination angles and their practical abilities, this paper investigates eight temporal and spatial features for characterizing angles of both artificial defects and RCF cracks under the influence of changing pocket lengths. Section II introduces the theoretical background of ECPT, zero-lag filtering, and the design of the crack shape reconstructor. In Section III, the experimental results on man-made angular slots and real RCF cracks are discussed and compared. Section IV gives the conclusion and future work.

\section{THEORETICAL BACKGROUND}

\section{A. Induction Heating of ECPT}

ECPT combines induction heating and heat diffusion processes. In brief, the heat diffusion equation in three-dimensional Cartesian coordinate with an induction heating source is [25]

$$
\rho C_{p} \frac{\partial T}{\partial t}=\lambda\left(\frac{\partial^{2} T}{\partial x^{2}}+\frac{\partial^{2} T}{\partial y^{2}}+\frac{\partial^{2} T}{\partial z^{2}}\right)+q
$$


where, $\rho, C_{\mathrm{p}}$, and $\lambda$ are the volumetric mass density, specific heat capacity, and thermal conductivity, respectively. $q\left(\mathrm{~W} / \mathrm{m}^{3}\right)$ is the power density of Joule heating. During the heating stage, $q$ can be considered as a constant having the form of

$$
q(x, y, z, t)=\frac{1}{\sigma(x, y, z)}|J(x, y, z)|^{2}
$$

where, $\sigma$ and $J$ are the electrical conductivity and electric current density, respectively.

Eq. (2) shows that $q$ at a specific position depends on both $\sigma$ and $J$. The existence of a defect will change $\sigma$ and $J$, which contribute to different $q$ values at its vicinity. Additionally, the defective area also has different $\rho, C_{\mathrm{p}}$, and $\lambda$. Taken together, these changes will influence Eq. (1) and a defect is detected by an abnormal thermal distribution or an abnormal transient thermal response. ECPT exploits both induction heating and heat diffusion. At the start of the heating stage, induction/Joule heating plays a dominant role with its advantage of surface flaw detection. In the later heating and cooling stages, heat diffusion takes over with the benefit of detecting subsurface and deeper flaws. Additionally, the induction coil, excitation parameters (heating pulse duration, frequency, and intensity), and the morphological parameters of defects (length, width, orientation, etc.) also lead to different thermal responses and distributions. Thus, thermal features based on various temporal-domain responses and spatial-domain distributions can be used for defect detection and characterization.

\section{B. Zero-lag Filtering for Temporal Thermal Response}

Features proposed in this work depend strongly on the calculation time slot. Commonly-used filters will introduce lag and then affect the time slot setting. To accurately retain key feature points, e.g., the maximum response time, in the filtered data, a zero-lag filter based on the Hamming window is proposed. The idea of zero-lag filtering is processing the input temporal response data in both the forward and reverse directions [26]. The filtered data has the characteristics of zero-phase distortion.

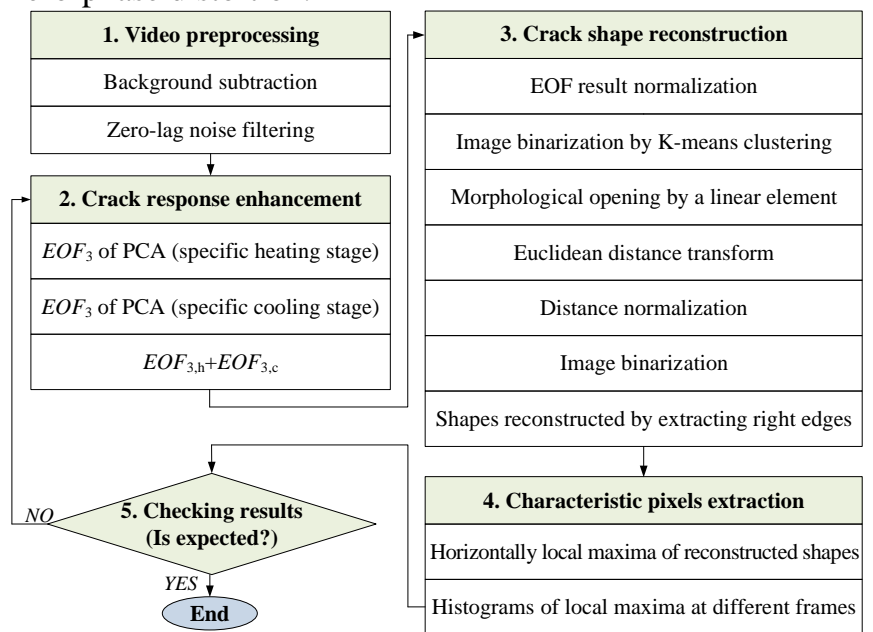

Fig. 1. Crack shape reconstructor broken down into five steps. Five steps are video preprocessing, crack response enhancement, crack shape reconstruction, characteristic pixels extraction, and checking final output results.
Specifically, an 11-point symmetric Hamming window-based FIR lowpass filter is used, which is defined as

$$
w[n]=a_{0}+\left(1-a_{0}\right) \cos \left(\frac{2 \pi n}{N}\right), \quad 0 \leq n \leq N
$$

where, $a_{0}$ is $25 / 46$, and $N$ is 10 .

This window can smooth signals without taking much computing time. Additionally, since the camera frame rate is $200 \mathrm{~Hz}$ and low frequencies are of much interest to crack detection, the cut-off frequency is set to $15 \mathrm{~Hz}$. The filter coefficients are given by

$$
x[n]=h[n] \cdot w[n]
$$

where, $h[n]$ is the unwindowed impulse response.

\section{Design of Crack Shape Reconstructor}

Due to the complex surface shapes of RCF cracks, the inclination angles cannot be directly characterized from the crack thermal responses/distributions. Thus, a crack shape reconstructor is proposed to first reconstruct the surface shapes of cracks and obtain the characteristic pixels around the reconstructed shapes. Fig. 1 shows the detailed reconstruction process and methods used in each step. In the video preprocessing step, background subtraction and the previously introduced zero-lag filtering are used. In the crack response enhancement step, the principal component analysis method is used to produce highly compressed descriptions of the thermal responses from both heating and cooling stages. By properly choosing an empirical orthogonal function (EOF), the underlying crack responses can be revealed and the third EOF $\left(E O F_{3}\right)$ is selected here. The further improved crack responses can be achieved by combining the $E O F_{3}$ from both heating and cooling stages, i.e., $E O F_{3, \mathrm{~h}}$ and $E O F_{3, \mathrm{c}}$. In this study, a $200 \mathrm{~ms}$ heating slot and a $300 \mathrm{~ms}$ cooling slot are found to achieve the expected results. In the crack shape reconstruction step, a set of image-processing procedures, including normalization, $\mathrm{K}$-means clustering-based binarization, linear element-based morphological opening, Euclidean distance transform, etc., are used to reconstruct the shapes of the cracks. Based on the reconstructed shapes, the next step finds characteristic pixels via the histograms of local maxima in the horizontal direction and at different frames. The results of this step then can be used to calculate the proposed features. The last step checks whether the results are physically reasonable. Here, the manual decision is made by comparing with the optical image. If not, the process will return to the crack response enhancement step and update the EOFs using a new window/span of heating and cooling stages. A detailed discussion is given in Section III. D.

\section{EXPERIMENTAL STUDIES}

The experimental results from testing angular slots and real RCF cracks are discussed. Eight temporal and spatial features are extracted and their relations to the inclination angles are compared.

\section{A. ECPT Configuration}

Fig. 2(a) shows the ECPT configuration used for experimental studies. It has four units, a heating module with a 
planar rectangular coil, an infrared (IR) camera, a signal generator, and a computer. The heating module is EASYHEAT 224 from Cheltenham Induction Heating with the operational RMS current of $300 \mathrm{~A}$ and frequency of $262 \mathrm{kHz}$. IR camera is FLIR A655sc. This camera is based on an uncooled microbolometer with the infrared sensing wavelengths from 7.5 to $14.0 \mu \mathrm{m}$ and the NETD within $30 \mathrm{mK}$. The maximum 200 $\mathrm{Hz}$ frame rate is selected, which leads to a spatial resolution of $640 \times 120$ pixels. In addition, to capture the detailed transient-spatial thermal distributions when testing the RCF specimen, a $2.9 \times$ close-up lens is mounted onto the standard lens. The heating module and the IR camera can be synchronously triggered by the signal generator. The recording duration is around $1000 \mathrm{~ms}$ for all the IR videos.

\section{B. Specimens}

Fig. 3 shows one specimen used in the experimental studies. As shown in Fig. 3(a), it is made of AISI 1045 carbon steel with nine artificial angular slots $\left(S_{1}-S_{9}\right)$ to simulate idealized RCF cracks [20], [27], [28]. Table I gives its material properties. The layout of the slots is shown in Fig. 3(b) and Table II gives their parameters. They were cut by wire electric discharge machining with the wire diameter of $0.2 \mathrm{~mm}$.

Additionally, a selected RCF rail specimen is used to verify the proposed features. Fig. 4 shows this cut-off specimen from a PD3 $(60 \mathrm{~kg} / \mathrm{m})$ rail removed from service. Compared with the AISI 1045 carbon steel, its carbon level is slightly different within the range of $0.7 \%$ to $0.8 \%$ [29]. This specimen contains nine RCF cracks having different inclination angles and pocket lengths. Here, only eight RCF cracks, denoted as $C_{1}-C_{8}$ in Fig. 4(a), are selected. This is later explained in Section III. D. The inclination angles $(\hat{\theta})$ and pocket lengths $(L)$ are estimated visually from the side view profile and 3D profile obtained from X-ray computed tomography, as shown in Figs. 4(b)-(c). Note that these cracks have varying inclination angles along the $y$-direction as shown in the bottom subfigure of Fig. 4(c). The estimated angle $(\hat{\theta})$ is considered as the average value of 14 sections in the $y$-direction. The limited extent of the RCF specimen on the left hand side affects the normal thermal diffusion. A PD3 block is attached trying to let the trapped heat quickly diffuse during experiments.

The top faces of both specimens were uniformly sprayed with the black matt paint to increase the emissivity. A $7 \mathrm{~mm}$ lift-off distance between the bottom of the coil and the top faces of the specimens is maintained in all experiments, as shown in Figs. 2(b) and 4(a).

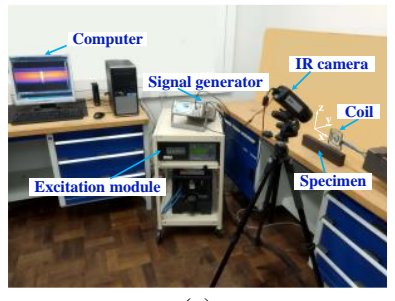

(a)

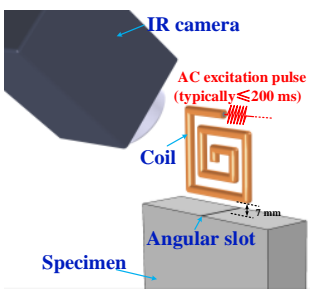

(b)
Fig. 2. ECPT configuration. (a) Layout of ECPT configuration. (b) Schematic diagram of ECPT configuration.

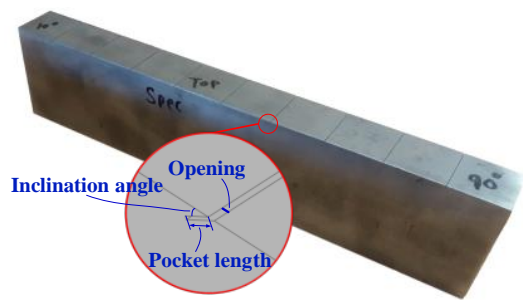

(a)



(b)
Fig. 3. Specimen with artificial angular slots. (a) Photo of the specimen. Its dimension is $(300 \times 30 \times 63) \mathrm{mm}^{3}$. Inclination angle, pocket length, and opening of a slot are illustrated in the zoom-in subfigure. (b) Locations of nine angular slots. $90^{\circ}$ simulates the vertical crack and the rest angles cover the common angle range of RCF cracks.

TABLE I

MATERIAL Properties OF MAN-MADE SPECIMEN [22]

\begin{tabular}{lll}
\hline Material properties & Value & Unit \\
\hline Density & $7.80 \times 10^{3}$ & $\mathrm{~kg} / \mathrm{m}^{3}$ \\
Specific heat capacity & $4.86 \times 10^{2}$ & $\mathrm{~J} /(\mathrm{kg} \cdot \mathrm{K})$ \\
Thermal conductivity & 49.8 & $\mathrm{~W} /(\mathrm{m} \cdot \mathrm{K})$ \\
Conductivity & $6.17 \times 10^{6}$ & $\mathrm{~S} / \mathrm{m}$ \\
\hline
\end{tabular}

\section{Feature Extraction and Comparison}

Six temporal features derived from the thermal responses and two spatial features derived from the thermal distributions are discussed in detail. The relations between features and the inclination angle are compared by three criteria, $R^{2}$, absolute slope, 2-norm NR. It is noteworthy that a well-fitted relation is described by a high $R^{2}$ and a small 2-norm NR. To eliminate the effects of different time slots or heating pulses, the residual values are normalized into the range of $(-1,1)$. Under each time slots or heating pulse, 2-norm of these normalized residuals are denoted as 2-norm NR.

Before extracting these features, some reference locations and time slots are needed since these features are related to both the temporal and spatial domains. A basic step is to identify the pixel that attains the highest thermal rise.

Fig. 5(a) shows an example of the thermal distribution at the end of the $200 \mathrm{~ms}$ heating pulse when $S_{8}$ is tested. The pixel with the highest thermal response is marked as the maximum thermal pixel (white dot). It is located at the $55^{\text {th }}$ row and the $93^{\text {rd }}$ column. However, this location changes at different frames due to measurement errors and uncertainty. Similar to previous work [22], the characteristic location of the maximum thermal pixel is statistically found by repeated tests, as shown in Fig. 5(b). Following the same approach, the characteristic locations of other slots also can be obtained.

After obtaining this key location, its thermal response can be extracted from the raw infrared video, as shown in Fig. 6. By using the designed zero-lag filter, the filtered thermal response is also shown as the black-bold curve. Further, the corresponding first-order differential response can be obtained by sliding the filtered thermal response. By only considering the rate of change of the thermal response, the absolute value of the first-order differential response is also plotted as the black-bold-dotted curve in this figure. Note that all the curves in Fig. 6 are normalized to $(0,1)$ or $(-1,1)$.

In this work, two periods, i.e., the later heating stage (shaded in light red), and the whole cooling stage (shaded in light blue), are utilized. The reason for using these two stages instead of the 
early heating stage is that even though the video recording and the pulse excitation are synchronized, a varying lag of the excitation still exists, as shown in the subfigure. In this case, the time lag is around $5 \mathrm{~ms}$ (one frame). Thus, a better way is to find the peak time (of the thermal response or the first-order differential response) first and then use time slots from its either side for further extracting different features.

Based on the maximum thermal pixel, the average thermal response of 21 pixels is calculated. The 21 pixels are in the same column but different rows, and the maximum thermal pixel is in the middle of them. Three repeated tests are conducted and their average value is used. After calculation, Figs. 7(a) and (b) show the normalized thermal responses and the first-order differential responses for $S_{1}-S_{9}$, respectively.

\section{1) Area-Based Features}

Based on the time slots of both the later heating and the cooling stages, the areas under the curves in Fig. 7 can be obtained. Figs. 8(a) and (b) give the values from the thermal responses and the first-order differential responses, respectively. They are denoted as $S U M_{\mathrm{T}}$ and $S U M_{\text {Diff1 }}$. It is clear that both $S U M_{\mathrm{T}}$ and $S U M_{\text {Diff1 }}$ increase with the time slot and they present the overall decreasing relations to the inclination angle. Note that all the feature values of $S_{9}$ (vertical slot), are excluded from the fitting data. From Fig. 8(a), the $800 \mathrm{~ms}$ time slot leads to the largest decreasing rate (slope). However, by selecting this time slot, the relation between $S U M_{\mathrm{T}}$ and the inclination angle is non-monotonic, as shown by the "+" sign on the value when the slot with the $10^{\circ}$ inclination angle is tested. This is also shown in the $S U M_{\mathrm{T}}$ row of Table III. On the other hand, by selecting the $100 \mathrm{~ms}$ or $150 \mathrm{~ms}$ time slot, even if the absolute value of the slope is less than $0.5, S U M_{\mathrm{T}}$ presents the monotonic relation to the inclination angle with a higher $R^{2}$ and a smaller 2-norm NR. From Fig. 8(b), both the $200 \mathrm{~ms}$ and $800 \mathrm{~ms}$ time slots contribute to the monotonically decreasing relations (all the $S U M_{\text {Diff1 }}$ values are marked with “-” sign). These two time slots give smaller 2-norm NR and the latter also leads to a larger absolute slope, as shown in the $S U M_{\text {Diff1 }}$ row of Table III. Comparing the $S U M_{\mathrm{T}}$ and $S U M_{\text {Diff1 }}$ rows of Table III, it shows that by using the $800 \mathrm{~ms}$ time slot, $S U M_{\text {Diff1 }}$ shows the best monotonically linear relation to the inclination angle with the $R^{2}$ and absolute slope value of $98.6 \%$ and 2.12 .

\section{2) Skewness-Based Features}

The characteristics of the thermal responses and their first-order differential responses in Fig. 7 can also be measured by some statistical values. The skewness [30] is used to measure the asymmetry of the histogram derived from either the thermal or first-order differential response. It can be assumed that slots with different inclination angles have individual thermal responses and further affect skewness values. By using different time slots, Figs. 8(c) and (d) show the skewness values from the thermal responses and first-order differential responses (denoted as $S_{\mathrm{T}}$ and $S_{\text {Diff1 } 1}$ ), respectively. The linear fitting relation can be improved by increasing the calculation time slot. From Fig. 8(c), none of the five time slots give a monotonic relation. On the other hand, in Fig. 8(d), only by using the $800 \mathrm{~ms}$ time slot, $S_{\text {Diff1 }}$ shows the monotonic relation to the inclination angle (all the values are marked with “+” sign). By comparing the $S_{\mathrm{T}}$ and $S_{\text {Diff1 }}$ rows of Table III, it shows that by choosing the $800 \mathrm{~ms}$ time slot, $S_{\mathrm{T}}$ has a higher $R^{2}$ value and a larger absolute slope value, but this relation is not monotonic. Although $S_{\text {Diff } 1}$ has a lower $R^{2}$ value and a smaller absolute slope, it still shows the monotonically increasing relation to the inclination angle.

\section{3) Kurtosis-Based Features}

Similarly, the kurtosis [30] is used here to measure whether the histograms from the thermal and first-order differential responses are heavy-tailed or light-tailed. Figs. 8(e) and (f) present the kurtosis values from the thermal responses and first-order differential responses (denoted as $K_{\mathrm{T}}$ and $K_{\text {Diff1 }}$ ), respectively. Both $K_{\mathrm{T}}$ and $K_{\text {Diff1 }}$ show better linear relations upon increasing the calculation time slot. Fig. 8(e) shows that only by using the $800 \mathrm{~ms}$ time slot, $K_{\mathrm{T}}$ shows the monotonic relation to the inclination angle (marked with "+" sign). From Fig. 8(f), none of the five time slots can give a monotonic relation. Comparing the $K_{\mathrm{T}}$ and $K_{\text {Diff1 }}$ rows of Table III, by choosing the $800 \mathrm{~ms}$ time slot, $K_{\mathrm{T}}$ shows the best monotonically linear relation to the inclination angle with the $R^{2}$, absolute slope, and 2-norm NR of $97.5 \%, 0.45$, and 1.84, respectively.

TABLE II

GEOMETRIC PARAMETERS OF ARTIFICIAL ANGULAR SLOTS

\begin{tabular}{|c|c|c|c|c|c|c|c|c|c|c|}
\hline Geometric parameter & Value & & & & & & & & & Unit \\
\hline & $S_{1}$ & $S_{2}$ & $S_{3}$ & $S_{4}$ & $S_{5}$ & $S_{6}$ & $S_{7}$ & $S_{8}$ & $S_{9}$ & \\
\hline Inclination angle of the slot & 10 & 15 & 20 & 25 & 30 & 35 & 40 & 45 & 90 & $\circ$ \\
\hline Pocket length of the $\operatorname{slot}^{* 1}$ & 0.38 & 0.45 & 0.81 & 0.90 & 0.88 & 1.03 & 0.91 & 1.06 & 1.32 & $\mathrm{~mm}$ \\
\hline Opening of the slot ${ }^{* 2}$ & 1.13 & 0.85 & 0.66 & 0.51 & 0.46 & 0.48 & 0.44 & 0.43 & 0.37 & $\mathrm{~mm}$ \\
\hline
\end{tabular}

${ }^{* 1}$ : Cut with different lengths and all within $1.5 \mathrm{~mm}$ to simulate initial-stage cracks; ${ }^{* 2}$ : Due to the inclination angle influence.

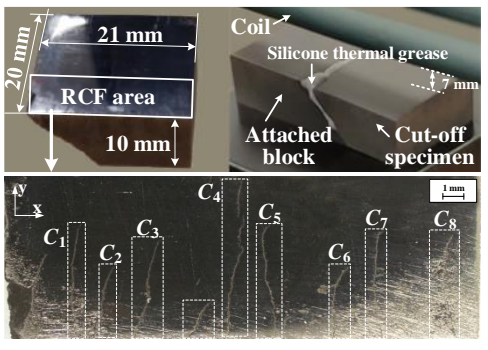

(a)

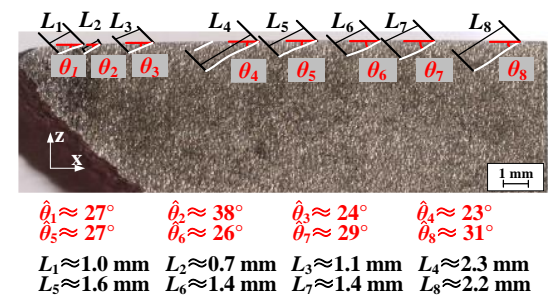

(b)

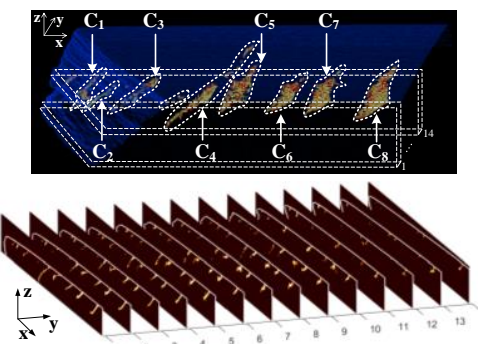

(c)

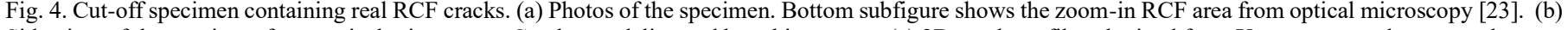
Side view of the specimen from optical microscopy. Cracks are delineated by white curves. (c) $3 \mathrm{D}$ crack profiles obtained from X-ray computed tomography. 


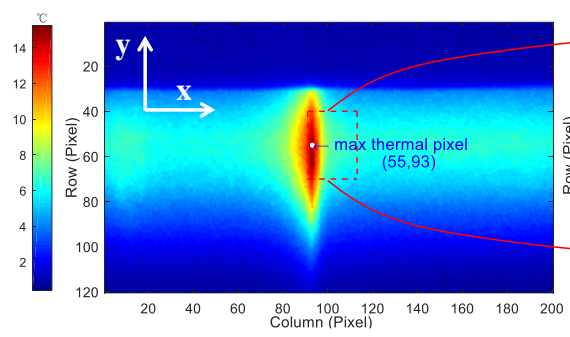

(a)

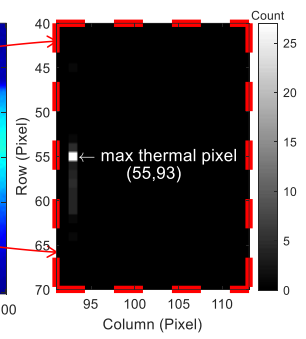

(b)
Fig. 5. ROI of slot vicinity and characteristic location of the maximum thermal pixel. (a) Thermal distribution of slot vicinity at $200 \mathrm{~ms}$ when $S_{8}$ is tested. The characteristic (maximum) thermal pixel is marked as the white dot. (b) Counts of the maximum pixels' locations by repeated tests.

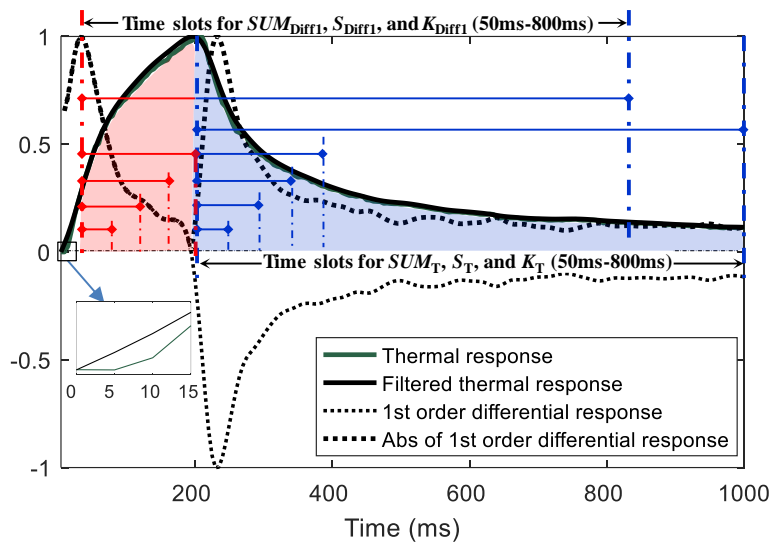

Fig. 6. Normalized thermal response and first-order differential response of the maximum thermal pixel when $S_{8}$ is tested.

\section{4) Spatial Skewness- and Kurtosis-Based Features}

Two spatial features, i.e., spatial skewness- and kurtosis-based features, are further examined. These two features are derived from the thermal distribution at the end of the heating stage and denoted as $S S_{\mathrm{T}}$ and $S K_{\mathrm{T}}$. The detailed calculation process can be found in [22], the only difference is that the thermal responses of the pixels are preprocessed by zero-lag filtering.

Figs. 9(a) and (b) show the $S S_{\mathrm{T}}$ and $S K_{\mathrm{T}}$ values under five different pulse durations, respectively. It can be seen that both $S S_{\mathrm{T}}$ and $S K_{\mathrm{T}}$ decrease with increasing pulse duration and that they exhibit increasing relations to the inclination angle. Additionally, Table IV shows that $S S_{\mathrm{T}}$ and $S K_{\mathrm{T}}$ have more positive correlations to the inclination angle under longer pulse durations. However, none of the five pulse durations give a monotonic relation. Comparing the $S S_{\mathrm{T}}$ and $S K_{\mathrm{T}}$ rows, it shows that by choosing the $200 \mathrm{~ms}$ pulse duration, $S S_{\mathrm{T}}$ has a better fitting relation to the inclination angle with a higher $R^{2}$ value of $87.3 \%$ and a smaller 2-norm NR of 1.73 .

\section{5) Discussion}

From the above comparisons, $S U M_{\mathrm{T}}, S U M_{\text {Diffl }}, S_{\text {Diffl }}$, and $K_{\mathrm{T}}$ show monotonic relations for longer time slots. Of these four features, $S U M_{\text {Diffl }}$ has the best linear relation to the inclination angle upon using the $800 \mathrm{~ms}$ time slot. Additionally, it can be concluded that by increasing the time slot or pulse duration, all eight features obtain larger absolute slopes in their relations to the inclination angle. The reason is that for temporal features, by increasing the time slot, any differences from thermal responses (or first-order differential responses) caused by the inclination angle changing are enlarged, as shown in Fig. 7. Particularly, for the three temporal features from the first-order differential response ( $S U M_{\text {Diff1 }}, S_{\text {Diffl }}$, and $\left.K_{\text {Diff1 }}\right)$, their absolute slopes are much larger upon choosing the $800 \mathrm{~ms}$ time slot. It is because this time slot includes both the contributions from heating and cooling stages. The above enlargement has two effects. One aspect is that the area-based features are enhanced by the accumulated differences. Another aspect is that the asymmetry and the tailed degree of the histogram from either the thermal or first-order differential response is also enhanced. For the spatial features, although a longer pulse duration reduces both the skewness and kurtosis values (because it leads to a longer heat diffusion time which smooths the spatial distribution), it increases the spatial contrast and stability. Thus, a better fitting relation with a higher slope value can be obtained.

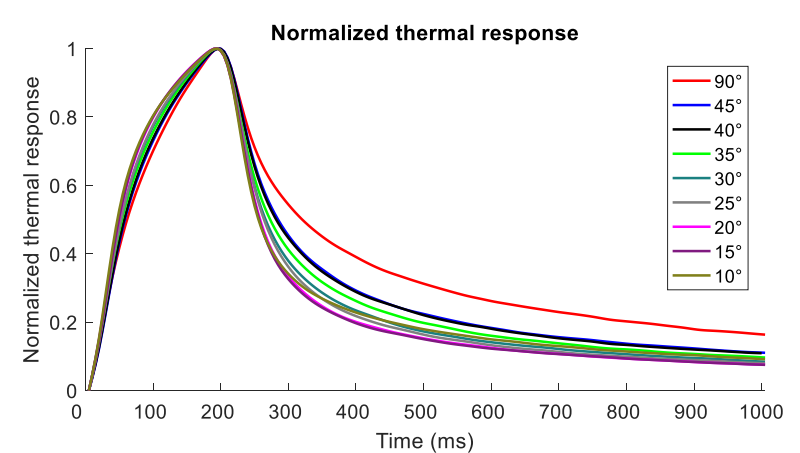

(a)

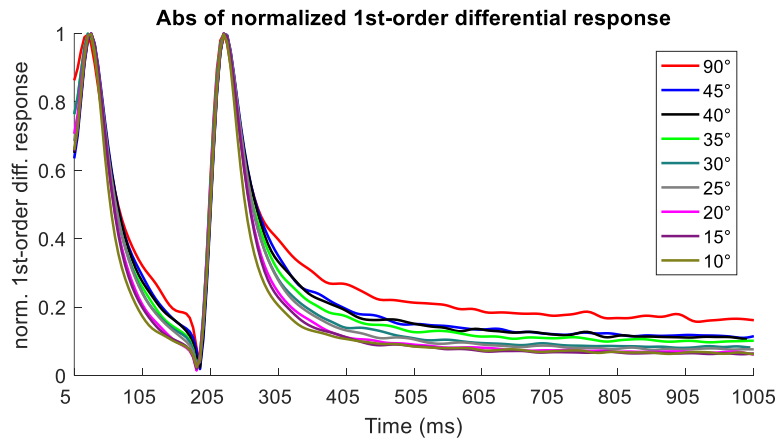

(b)

Fig. 7. Normalized thermal responses and the corresponding first-order differential responses from the defective areas of slots having different inclination angles. (a) Normalized thermal responses. (b) Normalized absolute first-order differential responses. 


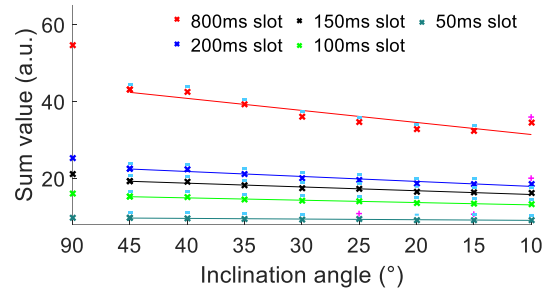

(a)



(d)

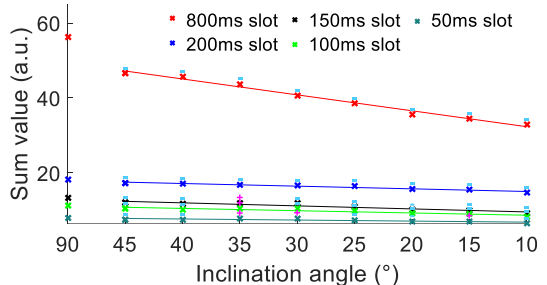

(b)

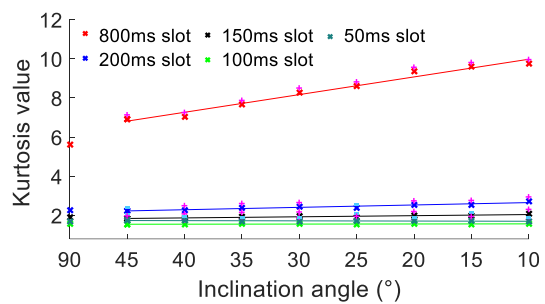

(e)

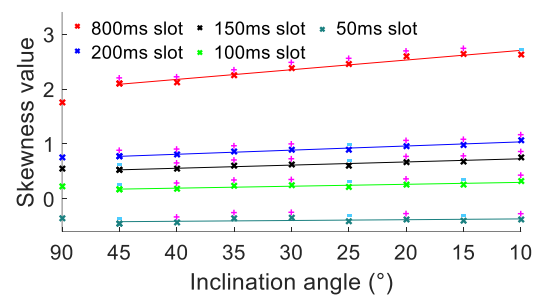

(c)

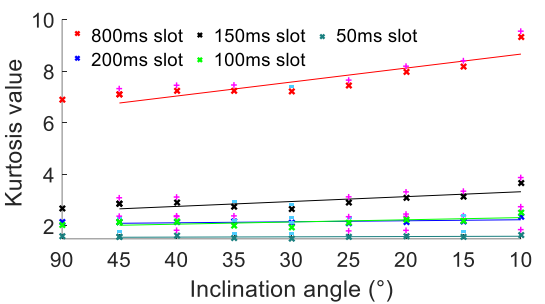

(f)

Fig. 8. Area-based $\left(S U M_{\mathrm{T}}\right.$ and $\left.S U M_{\text {Diffl }}\right)$, skewness-based $\left(S_{\mathrm{T}}\right.$ and $\left.S_{\text {Diffl }}\right)$, and kurtosis-based features $\left(K_{\mathrm{T}}\right.$ and $\left.K_{\text {Diffl }}\right)$ vs. inclination angle. All the values are calculated by using five different time slots $(50,100,150,200$, and $800 \mathrm{~ms})$. Solid lines show the linear fitting relations. "+" and "-" signs marked on the top of feature points show the increase or decrease between two neighbor points from left to right. (a) and (b) show the $S U M_{\mathrm{T}}$ and $S U M_{\text {Difft }}$ vs. inclination angle, respectively. (c) and (d) show the $S_{\mathrm{T}}$ and $S_{\text {Diffl }}$ vs. inclination angle, respectively. (e) and (f) show the $K_{\mathrm{T}}$ and $K_{\text {Diffl }}$ vs. inclination angle, respectively.

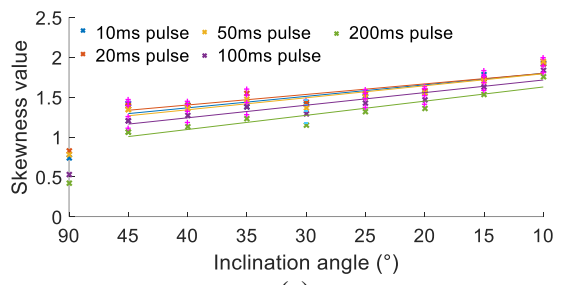

(a)

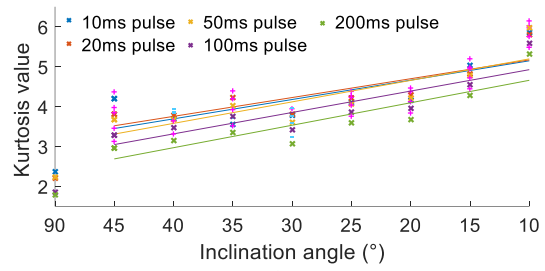

(b)

Fig. 9. Spatial skewness-based $\left(S S_{\mathrm{T}}\right)$ and kurtosis-based features $\left(S K_{\mathrm{T}}\right)$ vs. inclination angle. All the values are calculated under five different pulse/excitation durations $(10,20,50,100$, and $200 \mathrm{~ms})$. Solid lines show the linear fitting relations. "+" and "-" signs marked on the top of feature points show the increase or decrease between two neighbor points from left to right. (a) $S S_{\mathrm{T}}$ vs. inclination angle. (b) $S K_{\mathrm{T}}$ vs. inclination angle.

TABLE III

FITTING PARAMETERS UNDER DiFFERENT CALCULATION TIME SLOTS

\begin{tabular}{lllllll}
\hline & Time slot (ms) & 50 & 100 & 150 & 200 & 800 \\
\hline \multirow{4}{*}{$S U M_{\mathrm{T}}$} & $R^{2}$ & $92.7 \%$ & $97.2 \%$ & $95.6 \%$ & $93.7 \%$ & $82.9 \%$ \\
& Abs. slope & 0.09 & 0.30 & 0.49 & 0.65 & 1.57 \\
& 2-norm NR & 2.00 & 1.89 & 1.85 & 1.99 & 2.03 \\
& Mono-relation & $\times$ & $\sqrt{ }$ & $\sqrt{ }$ & $\times$ & $\times$ \\
\hline \multirow{5}{*}{$S U M_{\text {Diff1 }}$} & $R^{2}$ & $64.4 \%$ & $81.8 \%$ & $87.4 \%$ & $94.0 \%$ & $98.6 \%$ \\
& Abs. slope & 0.14 & 0.30 & 0.40 & 0.36 & 2.12 \\
& 2-norm NR & 1.90 & 1.98 & 1.96 & 1.67 & 1.80 \\
& Mono-relation & $\times$ & $\times$ & $\times$ & $\sqrt{ }$ & $\sqrt{ }$ \\
\hline \multirow{5}{*}{$S_{\mathrm{T}}$} & $R^{2}$ & $23.9 \%$ & $77.2 \%$ & $91.9 \%$ & $95.8 \%$ & $95.7 \%$ \\
& Abs. slope & 0.01 & 0.02 & 0.03 & 0.04 & 0.09 \\
& 2-norm NR & 2.14 & 2.03 & 1.78 & 1.71 & 1.73 \\
& Mono-relation & $\times$ & $\times$ & $\times$ & $\times$ & $\times$ \\
\hline \multirow{5}{*}{$S_{\text {Diff1 }}$} & $R^{2}$ & $23.6 \%$ & $44.9 \%$ & $66.9 \%$ & $72.0 \%$ & $83.4 \%$ \\
& Abs. slope & 0.02 & 0.03 & 0.04 & 0.02 & 0.06 \\
& 2-norm NR & 1.88 & 1.88 & 1.79 & 1.77 & 1.73 \\
& Mono-relation & $\times$ & $\times$ & $\times$ & $\times$ & $\sqrt{ }$ \\
\hline
\end{tabular}

\begin{tabular}{lllllll}
\hline \multirow{4}{*}{$K_{\mathrm{T}}$} & $R^{2}$ & $24.8 \%$ & $13.2 \%$ & $80.7 \%$ & $90.2 \%$ & $97.5 \%$ \\
& Abs. slope & 0.01 & 0 & 0.03 & 0.06 & 0.45 \\
& 2-norm NR & 2.29 & 2.23 & 1.90 & 1.71 & 1.84 \\
& Mono-relation & $\times$ & $\times$ & $\times$ & $\times$ & $\sqrt{ }$ \\
\hline \multirow{4}{*}{$K_{\text {Diff1 }}$} & $R^{2}$ & $10.0 \%$ & $36.5 \%$ & $55.3 \%$ & $36.8 \%$ & $75.9 \%$ \\
& Abs. slope & 0.01 & 0.04 & 0.09 & 0.02 & 0.27 \\
& 2-norm NR & 2.02 & 1.76 & 1.76 & 1.91 & 1.94 \\
& Mono-relation & $\times$ & $\times$ & $\times$ & $\times$ & $\times$ \\
\hline
\end{tabular}

TABLE IV

FitTing PARAmeters UNDER DifFerent HeAting PUlse DuRATIONS

\begin{tabular}{|c|c|c|c|c|c|c|}
\hline & Pulse duration (ms) & 10 & 20 & 50 & 100 & 200 \\
\hline \multirow{4}{*}{$S S_{\mathrm{T}}$} & $R^{2}$ & $77.4 \%$ & $80.2 \%$ & $79.4 \%$ & $85.5 \%$ & $87.3 \%$ \\
\hline & Abs. slope & 0.07 & 0.07 & 0.08 & 0.08 & 0.09 \\
\hline & 2-norm NR & 2.23 & 1.92 & 1.79 & 1.81 & 1.73 \\
\hline & Mono-relation & $x$ & $x$ & $x$ & $x$ & $x$ \\
\hline \multirow{4}{*}{$S K_{\mathrm{T}}$} & $R^{2}$ & $58.7 \%$ & $71.0 \%$ & $68.8 \%$ & $75.0 \%$ & $76.9 \%$ \\
\hline & Abs. slope & 0.24 & 0.24 & 0.27 & 0.27 & 0.28 \\
\hline & 2-norm NR & 2.17 & 1.95 & 1.93 & 1.91 & 1.86 \\
\hline & Mono-relation & $x$ & $x$ & $x$ & $x$ & $x$ \\
\hline
\end{tabular}

\section{Results Verification}

To verify the capabilities of the above-proposed features, they are applied to characterize real RCF cracks. Using the same ECPT configuration, the thermal distribution after a 200 $\mathrm{ms}$ heating pulse is shown in the bottom subfigure of Fig. 10(a). The red-dashed boxes show the relative positions of ROIs from optical and infrared images. Note that their viewing angles are different. The former is almost vertical and the latter is around $40^{\circ}$-angled to avoid the coil blocking the view. Via the crack shape reconstructor, the output of each step is shown in Figs. 10(b)-(d), respectively. By comparing the bottom subfigures of Figs. 10(a) and (b), it is evident that the PCA-based analysis can effectively extract and enhance the defective areas. As previously mentioned, this study only focuses on eight RCF cracks (denoted as $C_{1}-C_{8}$ ) and the crack to the left of $C_{4}$ is excluded. In Fig. 10(b), the response of this crack is not obvious. The reason is that this crack locates at the bottom edge of the specimen, which is outside the main heating area of the coil and produces a much weaker thermal response than other cracks. 
Consequently, in the crack shape reconstruction step, this crack disappears from the reconstructed results, as shown in the bottom of Fig. 10(c). It is noteworthy that although this crack is missed in this study, an optimized coil design, e.g., using a larger diameter or multiple turns, can easily address this problem. As in Section III. C., the maximum thermal pixels around the reconstructed shapes are needed as the base points to calculate the proposed features. Similarly, these locations are changing at different frames due to measurement errors and uncertainty. The locations of these maximum thermal pixels for the eight cracks are obtained by finding horizontally local maxima around the reconstructed shapes and their histograms at different frames, as shown in Fig. 10(d).

Based on these characteristic pixels, it is easy to calculate all eight features for different time slots and pulse durations. The results are shown in Fig. 11. It can be seen that all features show the same trends as the ones discussed in Section III. C. Table V gives the fitting parameters and monotonic relation checks. Note that only the best fitting relation of each feature is presented. Specifically, $S U M_{\mathrm{T}}, S U M_{\text {Diff1 }}, S_{\mathrm{T}}, S_{\text {Diffl }}, K_{\mathrm{T}}, K_{\text {Diff1 }}$ are calculated by using the $800 \mathrm{~ms}$ time slot, and $S S_{\mathrm{T}}, S K_{\mathrm{T}}$ are calculated by using the $200 \mathrm{~ms}$ pulse duration. Among different features, $S U M_{\mathrm{T}}, S U M_{\text {Diff1 }}, S_{\text {Diffl }}, K_{\mathrm{T}}$, and $K_{\text {Diffl }}$ gets higher $R^{2}$ values. Furthermore, $S U M_{\mathrm{T}}, S U M_{\mathrm{Diff}}$, and $K_{\mathrm{T}}$ also give monotonic relations. Of these three features, $S U M_{\text {Diff1 }}$ presents the best linear relation to the inclination angle with the highest $R^{2}$, the largest absolute slope, and the smallest 2-norm NR. However, for $S S_{\mathrm{T}}$ and $S K_{\mathrm{T}}$, the $R^{2}$ values lie below $90 \%$. This suggests that it is difficult for the spatial skewness- and kurtosis-based features to characterize inclination angles. Taken together, the temporal area-based and the kurtosis-based features are more suitable for the inclination angle characterization when testing real RCF cracks.

Image binarization by K-means clustering

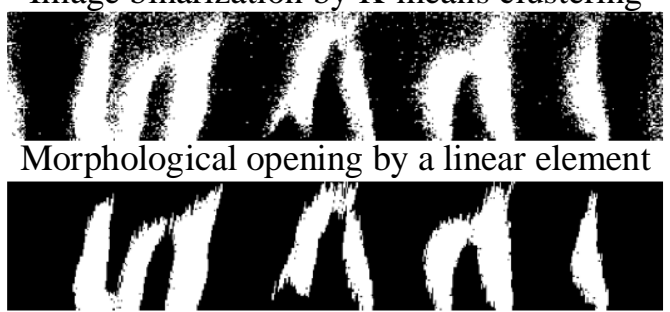

Distance transform, normalization, binarization

(a)

$\mathrm{EOF}_{3, \mathrm{~h}}$

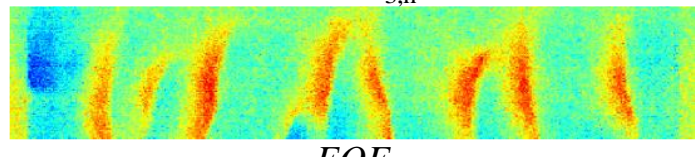

$E O F_{3, \mathrm{c}}$

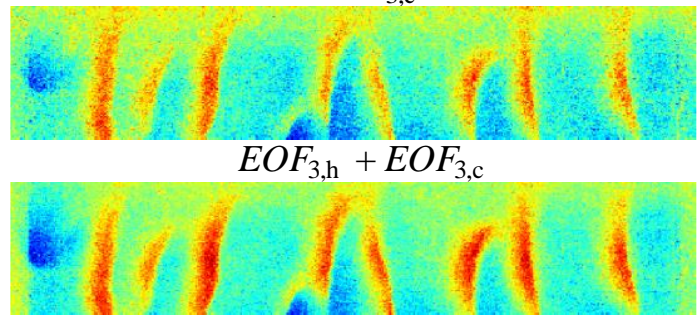

(b)

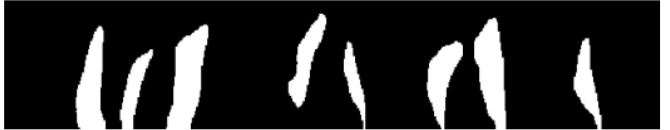

Shapes reconstructed by extracting right edges

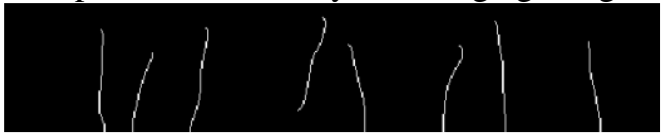

(c)

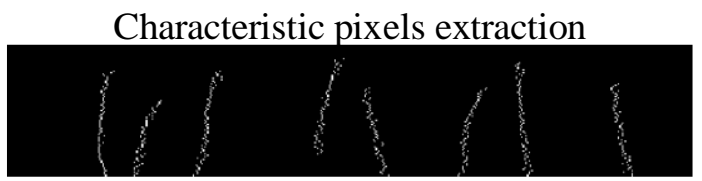

(d)

Fig. 10. Crack shapes and extracted characteristic pixels. (a) Preprocessed thermal frame after a $200 \mathrm{~ms}$ heating pulse by background subtraction, and zero-lag noise filtering. (b) Enhanced crack responses by PCA. (c) Reconstructed crack shapes by a set of image-processing procedures. (d) Extracted characteristic pixels by finding local maxima around the reconstructed shapes and their histograms at different frames.

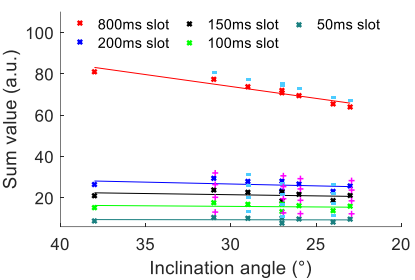

(a)

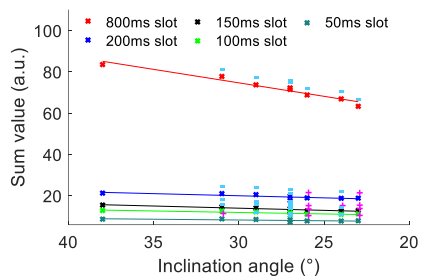

(b)

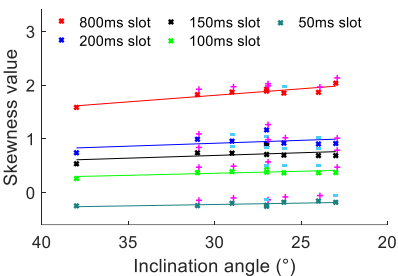

(c)

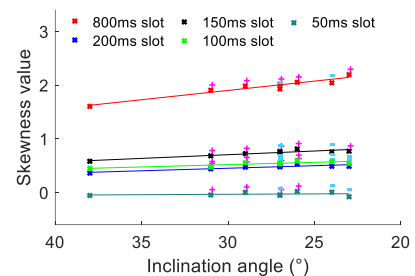

(d) 


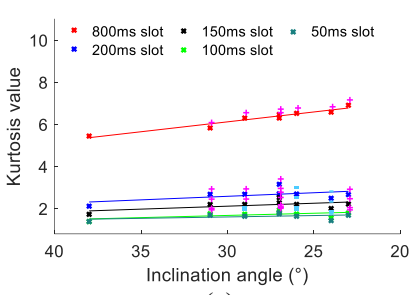

(e)

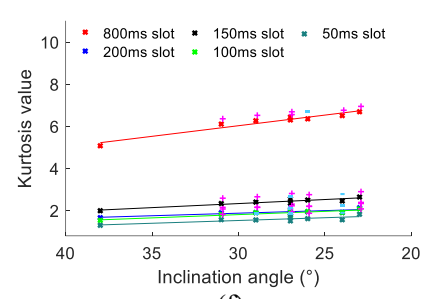

(f)

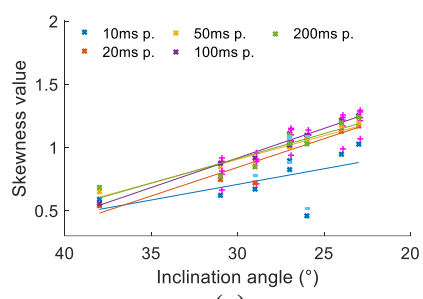

(g)

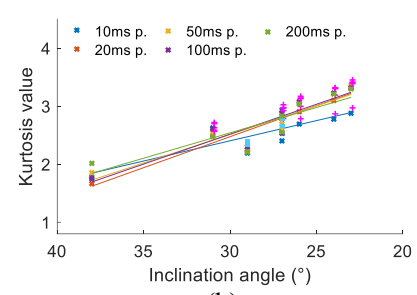

(h)

Fig. 11. $S U M_{\mathrm{T}}, S U M_{\text {Diffl }}, S_{\mathrm{T}}, S_{\text {Diffl }}, K_{\mathrm{T}}, K_{\mathrm{Diffl}}, S S_{\mathrm{T}}$, and $S K_{\mathrm{T}}$ vs. inclination angle. Temporal features are calculated by using five different time slots (50, 100, 150, 200, and $800 \mathrm{~ms})$. Solid lines show the linear fitting relations. Spatial features are calculated under five different pulse durations $(10,20,50,100$, and $200 \mathrm{~ms})$. " + " and "-" marked on the top of feature points show the increase or decrease between two neighbor points from left to right. (a) and (b) show the $S U M_{\mathrm{T}}$ and $S U M_{\mathrm{Diff}} \mathrm{vS.}$ inclination angle, respectively. (c) and (d) show the $S_{\mathrm{T}}$ and $S_{\text {Diffl }}$ vs. inclination angle, respectively. (e) and (f) show the $K_{\mathrm{T}}$ and $K_{\mathrm{Diffl}}$ vs. inclination angle, respectively. (g) and (h) show $S S_{\mathrm{T}}$ and $S K_{\mathrm{T}}$ vs. inclination angle, respectively.

TABLE V

FITTING PARAMETERS OF DIFFERENT RELATIONS

\begin{tabular}{lllllllll}
\hline & $S U M_{\mathrm{T}}$ & $S U M_{\text {Diffl }}$ & $S_{\mathrm{T}}$ & $S_{\text {Diff1 }}$ & $K_{\mathrm{T}}$ & $K_{\text {Diff1 }}$ & $S S_{\mathrm{T}}$ & $S K_{\mathrm{T}}$ \\
\hline Time slot (ms) & 800 & 800 & 800 & 800 & 800 & 800 & - & - \\
Pulse duration (ms) & - & - & - & - & - & - & 200 & 200 \\
$R^{2}$ & $91.5 \%$ & $95.0 \%$ & $82.4 \%$ & $91.6 \%$ & $93.6 \%$ & $93.7 \%$ & $85.8 \%$ & $78.4 \%$ \\
Abs. slope & 1.15 & 1.31 & 0.02 & 0.03 & 0.09 & 0.10 & 0.04 & 0.09 \\
2-norm NR & 1.99 & 1.88 & 2.07 & 2.12 & 1.97 & 2.04 & 2.13 & 2.27 \\
Monotonic & $\sqrt{ }$ & $\sqrt{ }$ & $\times$ & $\times$ & $\sqrt{ }$ & $\times$ & $\times$ & $\times$ \\
\hline
\end{tabular}

manufacturing using thermal monitoring," Appl. Therm. Eng., vol. 31, pp.978-983, 2011.

\section{CONCLUSION}

In this paper, six temporal and two spatial features have been defined for characterizing inclination angles of RCF cracks by eddy current pulsed thermography. These features have been evaluated against idealized defects, as well as real RCF cracks. Results show that the relations of these features to the inclination angle are clearer when using longer time slots and pulse durations. When testing real RCF cracks, $S U M_{\mathrm{T}}, S U M_{\text {Diff1, }}$, and $K_{\mathrm{T}}$ are the most suited for the inclination angle characterization.

Future work will focus on comparisons with other NDT methods. Under more complex distributions of RCF cracks, the capabilities of the proposed features need to be further verified. Additionally, feature selection for characterizing other parameters of RCF cracks, such as surface length, horizontal angle, vertical depth, should also be investigated.

\section{REFERENCES}

[1] Train derailment at Hatfield: A final report by the independent investigation board, Office of Rail Regulation, London, U.K., 2006.

[2] M. O. Speidel, "Stress corrosion cracking of aluminum alloys," Metall. Trans. A, vol. 6, pp.631-651, 1975.

[3] H. C. Eden et al., "Influential microstructural changes on rolling contact fatigue crack initiation in pearlitic rail steels," Mater. Sci. Technol., vol. 21, pp.623-629, 2005.

[4] W. Zhong et al., "A study of rolling contact fatigue crack growth in U75V and U71Mn rails," Wear, vol. 271, pp.388-392, 2011.

[5] M. Ph. Papaelias, et al., "A review on non-destructive evaluation of rails: state-of-the-art and future development," Proceedings of the Institution of Mechanical Engineers, Part F: Journal of Rail and rapid transit, vol. 222, pp.367-384, 2008.

[6] E. E. Magel, Rolling contact fatigue: a comprehensive review. Office of Railroad Policy and Development, Federal Railroad Administration, United States, 2011.

[7] R. A. Osornio-Rios et al., "Recent Industrial Applications of Infrared Thermography: A Review," IEEE Trans. Ind. Inf, vol. 15, pp.615-625, 2018.

[8] C. Meola, "Infrared thermography of masonry structures," Infrared Phys. Technol., vol. 49, pp.228-233, 2007.

[9] M. Manana et al., "Field winding fault diagnosis in DC motors during
[10] S. Stipetic, M. Kovacic, Z. Hanic, and M. Vrazic, "Measurement of excitation winding temperature on synchronous generator in rotation using infrared thermography," IEEE Trans. Ind. Electron., vol. 59, pp.2288-2298, 2012.

[11] A. N. Huda and S. Taib, "Application of infrared thermography for predictive/preventive maintenance of thermal defect in electrical equipment," Appl. Therm. Eng., vol. 61, pp.220-227, 2013.

[12] Y. Hu et al., "Identifying PV module mismatch faults by a thermography-based temperature distribution analysis," IEEE Trans. Device Mater. Rel., vol. 14, pp.951-960, 2014.

[13] D. B. Durocher and D. Loucks, "Infrared windows applied in switchgear assemblies: Taking another look," IEEE Trans. Ind. Appl., vol. 51, pp.4868-4873, 2015.

[14] X. P. Maldague, "Introduction to NDT by active infrared thermography," Mater. Eval., vol. 60, pp.1060-1073, 2002.

[15] R. Usamentiaga et al., "Automated Dynamic Inspection Using Active Infrared Thermography," IEEE Trans. Ind. Inf., vol. 14, pp.5648-5657, 2018.

[16] S. Doshvarpassand et al., "An overview of corrosion defect characterization using active infrared thermography," Infrared Phys. Technol., vol. 96, pp.366-389, 2018.

[17] B. Weekes et al., "Eddy-current induced thermography-probability of detection study of small fatigue cracks in steel, titanium and nickel-based superalloy," NDT \& E Int., vol. 49, pp.47-56, 2012.

[18] B. Gao et al., "Variational bayesian sub-group adaptive sparse component extraction for diagnostic imaging system," IEEE Trans. Ind. Elec., vol. 65, pp.8142-8152, 2018.

[19] J. Wu et al., "DC-biased Magnetization Based Eddy Current Thermography for Subsurface Defect Detection." IEEE Trans. Ind. Inf., vol. 15, pp. 6252-6259, 2019.

[20] I. Z. Abidin et al., "Quantitative evaluation of angular defects by pulsed eddy current thermography," NDT \& E Int., vol. 43, pp.537-546, 2010.

[21] B. Oswald-Tranta, "Induction Thermography for Surface Crack Detection and Depth Determination," Appl. Sci., vol. 8, p.257, 2018.

[22] F. Liu et al., "Investigations for inclination angle characterization of angular defects using eddy current pulsed thermography," Infrared Phys. Technol, vol. 100, pp.73-81, 2019.

[23] J. Zhu et al., "Comparison Study of Different Features for Pocket Length Quantification of Angular Defects Using Eddy Current Pulsed Thermography," IEEE Trans. Instrum. Meas., vol. 68, pp.1373-1381, 2019.

[24] J. Zhu et al., "Probability of detection for eddy current pulsed thermography of angular defect quantification," IEEE Trans. Ind. Inf., vol. 14 , pp. 5658-5666, 2018.

[25] H. S. Carslaw and J. C. Jaeger, Conduction of heat in solids. Oxford: Oxford Science Publications, 1959.

[26] V. A. Oppenheim et al., Discrete-Time Signal Processing. New Jersey: 
Pearson Higher Education, 2010.

[27] G.L. Nicholson et al., "Modelling and experimental measurements of idealised and light-moderate RCF cracks in rails using an ACFM sensor," NDT \& E Int., vol. 44, pp.427-437, 2011.

[28] J. Peng et al., "Investigation into eddy current pulsed thermography for rolling contact fatigue detection and characterization," $N D T \& E$ Int., 74, pp.72-80, 2015.

[29] W. J. Wang et al., "Wear and damage transitions of wheel and rail materials under various contact conditions," Wear, vol. 362, pp.146-152, 2016.

[30] F. J. Madruga et al., "Infrared thermography processing based on higher-order statistics," NDT \& E Int., vol. 43, pp.661-666, 2010.

Junzhen Zhu (S'19) is currently working toward the Ph.D. degree at School of Engineering, Newcastle University, U.K.

His research interests include eddy current pulsed thermography and vibrothermography.

Philip John Withers received his degree and $\mathrm{PhD}$ at the University of Cambridge taking up a lectureship there immediately afterwards.

Since 1998 he has been at Manchester University where he is now the first Regius Professor of Materials and Chief Scientist of the Henry Royce Institute (HRI) for Advanced Materials. He is an expert of the performance and degradation of engineering materials and in particular has pioneered the use of large-scale neutron and synchrotron facilities to follow behaviors under in-service conditions in situ by diffraction and computed tomography (CT). Under his leadership, the HRI and the Henry Moseley X-ray Imaging Facility bring together electron and $\mathrm{X}$-ray facilities for the $3 \mathrm{D}$ imaging of materials across a range of scale.

Jianbo Wu (M'19) is currently an Associate Professor at Sichuan University. He received the B.Sc., M.Sc., and Ph.D. degrees from the School of Mechanical Science and Engineering, Huazhong University of Science and Technology, Wuhan, China, in 2009, 2010, and 2014, respectively.

His research interests include magnetic flux leakage testing, eddy current thermography and eddy current testing.

Feng Liu is currently an Associate Professor at Guizhou Institute of Technology. He received the B.Sc. degree from the School of Precision Instruments and Optoelectronics Engineering, Tianjin University, Tianjin, China, in 2001, and the M.Sc. and Ph.D. degrees from the School of Mechanical Engineering, Sichuan University, Chengdu, China, in 2010, and 2014, respectively.

His research interests include eddy current thermography and optical image processing.
Qiuji Yi is currently the Ph.D. candidate and research assistant with School of Engineering, Newcastle University, U.K. He is also the research fellow in MSCA-H2020 NDTonAIR project.

His research interests include NDT via eddy current stimulated thermography, pulse-compression thermography, laser ultrasound and electromagnetic finite element analysis.

Zijun Wang is currently an Associate Research Fellow at University of Electronic Science and Technology of China. She received the B.Sc., M.Sc., and Ph.D. degrees from the School of Electrical Engineering and Automation, Harbin Institute of Technology, Harbin, China, in 2003, 2005, and 2010, respectively.

Her research interests include NDT\&E, infrared image processing and infrared target recognition.

Gui Yun Tian (M'01-SM'03) received the B.Sc. degree in metrology and instrumentation and the M.Sc. degree in precision engineering from the University of Sichuan, Chengdu, China, in 1985 and 1988, respectively, and the Ph.D. degree in nondestructive testing from the University of Derby, Derby, U.K., in 1998.

From 2000 to 2006, he was a Lecturer, Senior Lecturer, Reader, Professor, and Head of the group of Systems Engineering, respectively, with the University of Huddersfield, Huddersfield, U.K. Since 2007, he has been with Newcastle University, UK, as a Chair Professor involved with Sensor Technologies. He is currently also an Adjunct Professor with the School of Automation Engineering, University of Electronic Science and Technology of China, Chengdu, China. $\mathrm{He}$ has also coordinated several research projects from the Engineering and Physical Sciences Research Council, Royal Academy of Engineering and FP7. He also has a good collaboration with leading industrial companies such as Airbus, Rolls Royce, BP, nPower, Network Rail, and TWI. 